\title{
MRI Findings in Tumefactive Demyelinating Lesions: A Systematic Review and Meta-Analysis
}

(D) C.H. Suh, DH.S. Kim, DS.C. Jung, DC.G. Choi, and DS.J. Kim

\begin{abstract}
BACKGROUND: Accurate diagnosis of tumefactive demyelinating lesions is clinically important to avoid unnecessary invasive biopsy or inappropriate treatment.
\end{abstract}

PURPOSE: We aimed to evaluate conventional and advanced MR imaging findings of tumefactive demyelinating lesions and determine the diagnostic performance of MR imaging for differentiating tumefactive demyelinating lesions from primary brain tumor.

DATA SOURCES: A systematic search of Ovid MEDLINE and EMBASE up to December 6, 2017, was conducted.

STUDY SELECTION: Original articles describing MR imaging findings in patients with tumefactive demyelinating lesions were selected.

DATA ANALYSIS: The pooled incidences of conventional MR imaging findings of tumefactive demyelinating lesions were obtained with the DerSimonian and Liard random-effects model. The pooled sensitivity and specificity of MR imaging for differentiating tumefactive demyelinating lesions from primary brain tumor were obtained using the bivariate random-effects model.

DATA SYNTHESIS: Nineteen eligible studies with 476 patients with tumefactive demyelinating lesions were included. The pooled incidence of open ring or incomplete rim enhancement was $35 \%(95 \% \mathrm{Cl}, 24 \%-47 \%)$, which was significantly higher than the incidence of closed ring or complete rim enhancement $(18 \%[95 \% \mathrm{Cl}, 11 \%-29 \%] ; P=.0281)$. The pooled incidences of $\mathrm{T} 2$ hypointense rim, absent or mild mass effect, and absent or mild perilesional edema were $48 \%, 67 \%$, and $57 \%$, respectively. On advanced MR imaging, tumefactive demyelinating lesions showed a high apparent diffusion coefficient, peripheral restricted diffusion, and low cerebral blood volume. The pooled sensitivity and specificity of MR imaging for differentiating tumefactive demyelinating lesions from primary brain tumor were $89 \%$ (95\% Cl, 82\%-93\%) and 94\% (95\% Cl, 89\%-97\%), respectively.

LIMITATIONS: Seventeen of 19 studies were retrospective studies.

CONCLUSIONS: Conventional MR imaging findings may help differentiate tumefactive demyelinating lesions from primary brain tumor, though further study is needed to determine the added value of advanced MR imaging.

ABBREVIATIONS: DSC = dynamic susceptibility-weighted contrast-enhanced imaging; PCNSL = primary central nervous system lymphoma; TDL = tumefactive demyelinating lesion

$\mathrm{T}$ umefactive demyelinating lesions (TDLs) are large (usually $>2 \mathrm{~cm}$ ) demyelinating brain lesions that mimic primary brain tumors, including primary central nervous system lymphoma (PCNSL) and high-grade glioma. ${ }^{1-3}$ Accurate diagnosis of

Received March 10, 2018; accepted after revision May 9.

From the Department of Radiology and Research Institute of Radiology, University of Ulsan College of Medicine, Asan Medical Center, Seoul, Republic of Korea.

This study was supported by a grant from the National R\&D Program for Cancer Control, Ministry of Health and Welfare, Republic of Korea (1720030).

Please address correspondence to Ho Sung Kim, MD, PhD, Department of Radiology and Research Institute of Radiology, University of Ulsan College of Medicine, Asan Medical Center, 86 Asanbyeongwon-Gil, Songpa-Gu, Seoul 138-736, Republic of Korea; e-mail: radhskim@gmail.com
TDL is clinically important to avoid unnecessary invasive biopsy or inappropriate treatment.

Several characteristic conventional MR imaging findings and advanced MR imaging techniques have been introduced for the diagnosis of TDLs. ${ }^{4-22}$ Conventional MR imaging findings, ${ }^{23}$ including open ring or incomplete rim enhancement, a T2 hypointense rim, absent or mild mass effect, and absent or mild perilesional edema, demonstrate variable frequencies. In addition, 1 review article classified TDLs into 4 different subtypes based on

\footnotetext{
- Indicates open access to non-subscribers at www.ajnr.org

Indicates article with supplemental on-line photo.

http://dx.doi.org/10.3174/ajnr.A5775
} 
the most prominent conventional MR imaging characteristics as follows: megacystic, Balò-like, infiltrative, and ringlike. ${ }^{24}$ Several studies have reported the use of advanced MR imaging techniques, including diffusion-weighted imaging, dynamic susceptibility-weighted contrast-enhanced imaging (DSC), MR spectroscopy, and diffusion tensor imaging. 5,8,10,12,14,15,18,19

To our knowledge, the MR imaging findings of TDLs and their diagnostic performance for differentiating TDLs from primary brain tumors have not yet been systematically reviewed. Therefore, we aimed to evaluate conventional and advanced MR imaging findings of TDLs and to determine the diagnostic performance of MR imaging for differentiating TDL from primary brain tumor.

\section{MATERIALS AND METHODS Search Methods and Study Selection}

A systematic search of the literature in MEDLINE and EMBASE was performed to identify published original articles describing MR imaging findings in patients with TDL. The search term combined synonyms of "TDL" and "MR imaging" as follows: ((tumefactive demyelinating lesion ${ }^{\star}$ ) OR (TDL) OR (atypical demyelination lesion) OR (Balò sclerosis)) AND ((MR imaging) OR (MR imaging) OR (MR imaging)). The data base was searched for literature published on or before April 21, 2018. The literature search was limited to English-language publications. The bibliographies of articles were explored to identify additional relevant articles.

\section{Data Extraction}

Conventional MR imaging findings and advanced MR imaging findings in patients with TDL and the diagnostic performance of MR imaging for differentiating TDL from primary brain tumor were extracted from the eligible articles. A TDL was defined as a large (usually $>2 \mathrm{~cm}$ ) demyelinating brain lesion mimicking a primary brain tumor. ${ }^{25,26}$ The conventional MR imaging findings of TDLs were recorded as the following: 1) an open ring or incomplete rim enhancement, 2) a closed ring or complete rim enhancement, 3) a T2 hypointense rim, 4) an absent or mild mass effect, and 5) absent or mild perilesional edema. Advanced MR imaging findings from the techniques of DWI, DSC, and MR spectroscopy were also recorded. Two-by-2 tables (true-positive, false-positive, false-negative, true-negative) for the determination of the diagnostic performance of MR imaging for differentiating TDLs from primary brain tumors were also constructed.

The following information was recorded from the selected studies: 1) the institution, the patient recruitment period, a retrospective or prospective design, consecutive or nonconsecutive patient enrollment, the reference standard, and the follow-up period; 2) the number of patients with TDLs, mean age, age range, and male-to-female ratio; 3 ) the magnetic field strength of the scanner, scanner manufacturer, scanner model, number of head coil channels, slice thickness, and advanced MR imaging techniques used, including DWI, DSC, and MR spectroscopy; and 4) the number of MR imaging readers, reader experience, and blindness to the reference standard.

Quality assessment was performed using the Quality Assessment of Diagnostic Accuracy Studies-2 (QUADAS-2) criteria. $^{27}$
The study selection, data extraction, and quality assessment were performed by 2 reviewers (C.H.S., H.S.K.) and were independently reviewed by a third reviewer (S.J.K.), in accordance with the Preferred Reporting Items for Systematic Reviews and MetaAnalyses guidelines. ${ }^{28}$

\section{Statistical Methods}

The pooled incidences for conventional MR imaging findings of TDL were obtained with the inverse variance method for calculating weights and the DerSimonian and Liard random-effects model. ${ }^{29-31}$ The difference between the incidences of open ring or incomplete rim enhancement versus closed ring or complete rim enhancement was evaluated using mixed-effects model meta-regression. Heterogeneity was evaluated using the inconsistency in$\operatorname{dex}\left(\mathrm{I}^{2}\right)$ test of Higgins et $\mathrm{al}^{32}$ with values of $>50 \%$ indicating substantial heterogeneity. Publication bias was visually assessed using a funnel plot, and the statistical significance was assessed using the Egger test. ${ }^{33}$ Meta-regression was performed to explain the effects of heterogeneity across the studies. We considered the following covariates: 1$)$ the number of patients with TDL ( $<15$ [median value of the included studies] versus $\geq 15$ ); 2 ) age (younger than 34.5 years [median value of the included studies] versus 34.5 years and older); 3 ) male-to-female ratio $(<0.76$ [median value of the included studies] versus $\geq 0.76$ ); 4) reference standard (histopathology only versus others); 5) magnetic field of the scanner ( $1.5 \mathrm{~T}$ versus $3 \mathrm{~T}$ ); and 6 ) slice thickness ( $<5 \mathrm{~mm}$ versus $\geq 5 \mathrm{~mm}$ ).

The pooled sensitivity and specificity and their 95\% confidence interval were obtained using the bivariate random-effects model. ${ }^{29-31}$ A hierarchic summary receiver operating characteristic curve with $95 \%$ confidence and prediction regions was obtained. Publication bias was assessed by the Deeks funnel plot, and the statistical significance was assessed by the Deeks asymmetry test. ${ }^{34}$

All statistical analyses were performed by 1 reviewer (C.H.S., with 5 years of experience in performing systematic reviews and meta-analysis) using the "metafor" and "mada" packages in R, Version 3.4.1 (http://www.r-project.org/) and the "metandi" and "MIDAS" modules in STATA 15.0 (StataCorp, College Station, Texas).

\section{RESULTS}

\section{Eligible Studies and Characteristics}

The search identified 195 articles. After removing 21 duplicated articles, we performed screening of the 174 remaining titles and abstracts. From these, a further 147 articles were excluded (Fig 1). Full-text reviews of the remaining 27 potentially eligible articles were performed, and a further 8 studies were excluded for the following reasons: no mention of the MR imaging findings of TDL $(n=4)$, partially overlapping patient cohorts $(n=3)$, and case series $(n=1)$. Finally, 19 eligible studies covering 476 patients with TDLs were included in the analyses. ${ }^{4-22}$

The Table lists the characteristics of the eligible studies published between 2001 and 2017. The mean age ranged from 10.4 to 42 years. One study was prospective in design, ${ }^{22} 17$ studies were retrospective, ${ }^{4-10,12-21}$ and 1 study did not mention the design. ${ }^{11}$ The clinical follow-up ranged from 9.6 months to 5 years. 


\section{Quality Assessment}

The quality of the 19 included studies was moderate, with $>4$ of the 7 domains being satisfied (On-line Fig 1). With regard to patient selection, 17 studies were considered to have an unclear risk of bias due to nonconsecutive enrollment. ${ }^{4,5,8-22}$ With regard to the reference standard, 5 studies were considered to have a high risk of bias because they only used clinical diagnosis for the reference standard. ${ }^{4,7,10,15,16}$ In the flow and timing domain, 5 studies had an unclear risk of bias because the follow-up interval was not reported, ${ }^{6,7,12,14,21}$ and 13 studies had a high risk because different reference standards were used in the study. ${ }^{4-6,8,11,12,14,16,18-22}$

\section{Incidence of Conventional MR Imaging Findings in Patients with TDL: Meta-Analysis}

First, we evaluated the pooled incidences of conventional MR imaging findings in patients with TDL (Fig 2). The pooled incidence of open ring or incomplete rim enhancement was $35 \%$ (95\% CI, 24\%-47\%), and the pooled incidence of closed ring or complete rim enhancement was $18 \%$ (95\% CI, 11\%$29 \%$ ), with the incidence of open ring or incomplete rim enhancement being significantly higher than that of closed ring or complete rim enhancement $(P=.028)$. The pooled incidence of a T2 hypointense rim was 48\% (95\% CI, 36\%-60\%), the pooled incidence of an absent or mild mass effect was $67 \%$ (95\% CI, 48\%-83\%), and the pooled incidence of absent or mild perilesional edema was $57 \%$ (95\% CI, 36\%-76\%).

There was no publication bias with respect to open ring or incomplete rim enhancement $(P=.81)$, though publication bias was noted with respect to closed ring or complete rim enhancement $(P<.001)$. Heterogeneity was observed for all conventional MR imaging findings $\left(\mathrm{I}^{2}=63 \%-80 \%\right)$. To explore the effects of heterogeneity, we conducted a meta-regression for open ring or incomplete rim enhancement. None of the variables, including the number of patients with TDL $(P=$ $.43)$, age $(P=.26)$, male-to-female ratio $(P=.24)$, reference standard $(P=$ $.51)$, magnetic field strength of the scanner $(P=.45)$, and slice thickness $(P=$ $.07)$, showed a statistically significant relationship.

FIG 1. Flow diagram for the literature-selection process.

\section{Characteristics of the eligible studies}

\begin{tabular}{|c|c|c|c|c|c|c|c|}
\hline Author (yr) & $\begin{array}{l}\text { Patients with } \\
\text { TDL (No.) }\end{array}$ & $\begin{array}{l}\text { Male } \\
\text { (No.) }\end{array}$ & $\begin{array}{c}\text { Female } \\
\text { (No.) }\end{array}$ & $\begin{array}{c}\text { Mean } \\
\text { Age (yr) }\end{array}$ & $\begin{array}{c}\text { Age } \\
\text { Range (yr) }\end{array}$ & $\begin{array}{c}\text { Duration of } \\
\text { Patient Recruitment }\end{array}$ & Institution \\
\hline Altintas et al $(2012)^{4}$ & 54 & 17 & 37 & 31.8 & $18-63$ & NA & 5 Medical centers, Turkey \\
\hline Cha et al $(2001)^{5}$ & 10 & 3 & 7 & 34.5 & $13-57$ & NA & New York University Medical Center \\
\hline Hiremath et al $(2017)^{6}$ & 14 & 10 & 4 & 37.5 & NA & 2011.1-2015.12 & $\begin{array}{l}\text { Sree Chitra Tirunal Institute for Medical Sciences } \\
\text { and Technology, India }\end{array}$ \\
\hline Jain et al $(2017)^{7}$ & 11 & 7 & 4 & 19.6 & $10-41$ & $2014.8-2017.3$ & Sawai Man Singh Medical College, India \\
\hline Kilic et al $(2013)^{8}$ & 25 & 8 & 17 & 29 & $15-56$ & 1993.2-2011.6 & Hacettepe University, Faculty of Medicine, Turkey \\
\hline Kim et al (2009) ${ }^{9}$ & 15 & 8 & 7 & 42 & $27-57$ & 1998.12-2005.12 & $\begin{array}{l}\text { Seoul National University Hospital and Samsung } \\
\text { Medical Center, Republic of Korea }\end{array}$ \\
\hline Kiriyama et al $(2011)^{10}$ & 12 & 6 & 6 & 27 (Median) & $17-64$ & 1993.1-2009.6 & Nara Medical University, Japan \\
\hline Kuan et al $(2013)^{11}$ & 12 & 1 & 11 & 41.5 & $16-62$ & 1985.1-2010.12 & Taipei Veterans General Hospital, Taiwan \\
\hline Lu et al $(2015)^{12}$ & 18 & 10 & 8 & NA & $22-66$ & $2007.4-2012.5$ & Asan Medical Center, Republic of Korea \\
\hline Lucchinetti et al $(2008)^{13}$ & 151 & NA & NA & NA & NA & 1987.9-2005.8 & $\begin{array}{l}\text { Mayo Clinic and Georg-August University, } \\
\text { Germany }\end{array}$ \\
\hline Mabray et al $(2015)^{14}$ & 24 & 10 & 14 & 35.1 & $16-53$ & $2002-2011$ & University of California at San Francisco \\
\hline Malhotra et al (2009) ${ }^{15}$ & 18 & 8 & 10 & 26 & $4-50$ & NA & $\begin{array}{l}\text { Chhatrapati Sahuji Maharaj Medical University } \\
\text { and Sanjay Gandhi Post Graduate Institute of } \\
\text { Medical Sciences, India }\end{array}$ \\
\hline Miron et al $(2013)^{16}$ & 10 & 2 & 8 & 26.8 & NA & 2007.1-2007.12 & Sheba Medical Center, Israel \\
\hline Qi et al $(2015)^{17}$ & 14 & 9 & 5 & 24 (Median) & $4-51$ & 2004.1-2009.1 & Beijing Tiantan Hospital, China \\
\hline Saini et al $(2011)^{18}$ & 18 & 10 & 8 & 31.8 & $10-61$ & 2001.1-2009.12 & $\begin{array}{l}\text { Sree Chitra Tirunal Institute for Medical Sciences } \\
\text { and Technology, India }\end{array}$ \\
\hline Sánchez et al $(2017)^{19}$ & 15 & 3 & 12 & 36 & NA & $2010.1-2017.2$ & Hospital Universitario de La Princesa, Spain \\
\hline Siri et al $(2015)^{20}$ & 16 & 5 & 11 & 35.7 & $20-65$ & NA & 10 Medical centers, France \\
\hline Toh et al $(2012)^{21}$ & 8 & 2 & 6 & 37.3 & $23-51$ & NA & National Yang-Ming University, Taiwan \\
\hline Yiu et al $(2014)^{22}$ & 31 & 14 & 17 & 10.4 & NA & 2004.9-2009.12 & 23 Medical centers, Canada \\
\hline
\end{tabular}

Note:-NA indicates not available. 
Study

Jain RS et al 2017
Kilic AK et al 2013
Kim DS et al 2009
Kiriyama T et al 2011
Lu SS et al 2015
Lucchinetti CF et al 2008
Mabray MC et al 2015
Malhotra HS et al 2009
Miron S et al 2013
Qi W et al 2015
Saini J et al 2011
Sanchez P et al 2017
Siri A et al 2015
Toh CH et al 2012
Yiu EM et al 2014

Yiu EM et al 2014

Heterom model

A

Study
Altintas A et al 2012
Kilic AK et al 2013
Kim DS et al 2009
Kiriyama T et al 2011
Lu SS et al 2015
Lucchinetti CF et al 2008
Malhotra HS et al 2009
Miron S et al 2013
Qi W et al 2015
Saini J et al 2011
Sanchez P et al 2017
Siri A et al 2015
Toh CH et al 2012
Yiu EM et al 2014
Random effects model

Random effects model B

\section{Study}

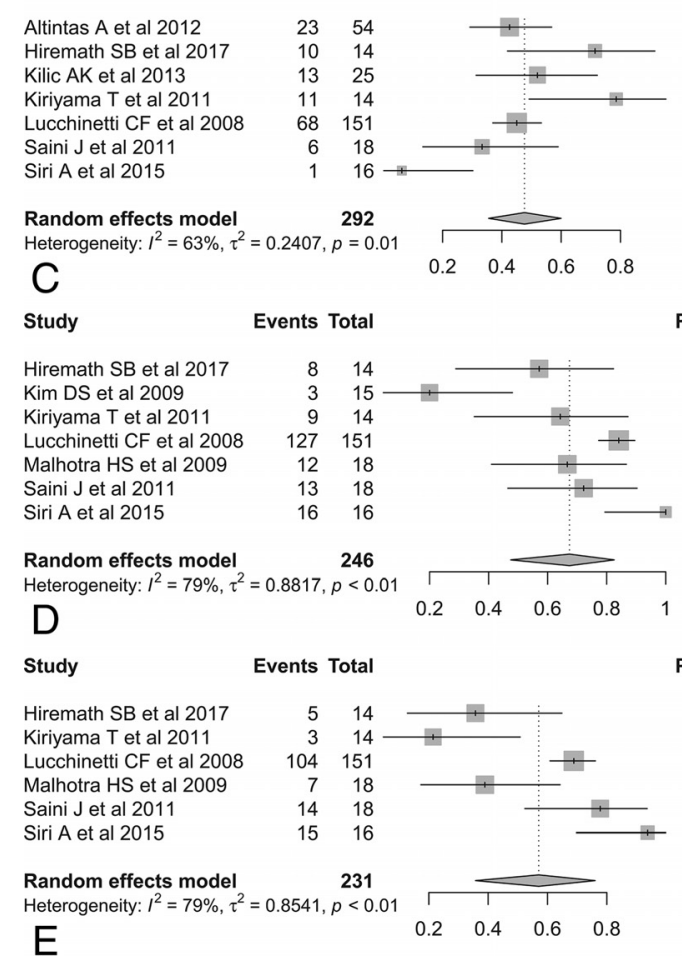

Events Total

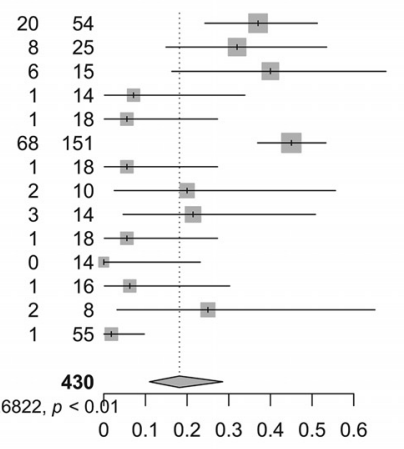

$\begin{array}{lllllll}0 & 0.1 & 0.2 & 0.3 & 0.4 & 0.5 & 0.6\end{array}$

\section{Events Total}

\section{Pr}

Proportion $\quad 95 \%-\mathrm{Cl}$ Weight

$0.37[0.24 ; 0.51] \quad 11.8 \%$ $0.32[0.15 ; 0.54] 10.4 \%$ $0.40[0.16 ; 0.68] \quad 9.3 \%$ $0.07[0.00 ; 0.34] \quad 5.1 \%$ $0.06[0.00 ; 0.27] \quad 5.2 \%$ $0.45[0.37 ; 0.53] \quad 12.7 \%$ $0.06[0.00 ; 0.27] \quad 5.2 \%$ $0.20[0.03 ; 0.50] \quad 6.0 \%$ $0.20[0.03,0.56] \quad 6.9 \%$ $0.21[0.05 ; 0.51] \quad 8.1 \%$ $0.06[0.00 ; 0.27] \quad 5.2 \%$ $0.00[0.00 ; 0.23] \quad 3.3 \%$ $0.06[0.00 ; 0.30] \quad 5.1 \%$ $0.25[0.03 ; 0.65] \quad 6.7 \%$ $0.02[0.00 ; 0.10] \quad 5.3 \%$

$0.18[0.11 ; 0.29] 100.0 \%$

\section{5\%-Cl Weight}

$0.43[0.29 ; 0.57] 20.5 \%$ $0.71[0.42 ; 0.92] \quad 11.0 \%$ $0.52[0.31 ; 0.72] 16.2 \%$ $0.79[0.49 ; 0.95] \quad 9.8 \%$ $0.45[0.37 ; 0.53] \quad 24.3 \%$ $0.33[0.13 ; 0.59] \quad 13.2 \%$ $0.06[0.00 ; 0.30] \quad 5.0 \%$

$0.48[0.36 ; 0.60] 100.0 \%$

\section{Proportion $\quad 95 \%-\mathrm{Cl}$ Weight}

$0.57[0.29 ; 0.82] 15.2 \%$ $0.20[0.04 ; 0.48] \quad 13.7 \%$ $0.64[0.35 ; 0.87] 14.9 \%$ $0.84[0.77 ; 0.90] 19.1 \%$ $0.67[0.41 ; 0.87] \quad 15.7 \%$ $0.72[0.47 ; 0.90] \quad 15.4 \%$ $1.00[0.79 ; 1.00] \quad 6.0 \%$ $0.67[0.48 ; 0.83] 100.0 \%$

Proportion $\quad 95 \%-\mathrm{Cl}$ Weight

$0.36[0.13 ; 0.65] \quad 17.0 \%$ $0.21[0.05 ; 0.51] 15.5 \%$ $0.69[0.61 ; 0.76] \quad 22.3 \%$ $0.39[0.17: 0.64] \quad 18.2 \%$ $0.78[0.52 ; 0.94] 16.8 \%$ $0.94[0.70 ; 1.00] 10.3 \%$

$0.57[0.36 ; 0.76] 100.0 \%$

FIG 2. Forest plots to show the pooled incidences of conventional MR imaging findings in patients with TDL: open ring or incomplete rim enhancement $(A)$, closed ring or complete rim enhancement $(B)$, T2 hypointense rim $(C)$, absent or mild mass effect $(D)$, and absent or mild perilesional edema (E). Numbers are estimates with $95 \%$ confidence intervals in parentheses.

\section{Advanced MR Imaging Findings in Patients with TDL}

On DWI, 2 studies demonstrated a significantly higher apparent diffusion coefficient for TDL than for PCNSL ${ }^{12,14}$ and high-grade glioma. ${ }^{14}$ Three studies also reported peripheral, restricted diffusion in TDLs. ${ }^{8,15,19,21}$ One study reported that TDLs showed higher intralesional hyperintensities on fractional anisotropy maps but lower perilesional fractional anisotropy values in the rim than highgrade gliomas. ${ }^{21}$ On perfusion imaging, 1 study demonstrated that TDLs showed a significantly lower relative cerebral blood volume than intracranial neoplasms, ${ }^{5}$ and 2 studies showed decreased CBV in TDLs on $\mathrm{MR}^{8,19}$ or CT perfusion. ${ }^{8}$ In addition, 1 study reported the presence of an intact vein traversing the TDL on DSC. ${ }^{5}$ For MR spectroscopy, several studies reported increased choline $e^{8,10,15,19}$ and decreased $N$-acetylaspartate, ${ }^{8,10,18,19}$ the presence of a lactate peak,,$^{8,15,18}$ and the presence of a glutamate/glutamine peak. ${ }^{15,18}$ The representative case studied by conventional and advanced MR imaging is shown in Fig 3.

\section{Individual Diagnostic Performance of MR Imaging for the Diagnosis of TDLs}

The diagnostic performance of conventional MR imaging for the diagnosis of TDLs was reported in 3 studies. ${ }^{6,9,14}$ By means of open ring or incomplete rim enhancement for the diagnosis of TDLs, the individual sensitivity showed substantial variation $(27 \%-71 \%)$, though the specificity was consistently high (98\%-100\%). ${ }^{9,14}$ In addition, 1 study using conventional MR imaging and CT criteria (less attenuation than cortical and basal ganglia gray matter) showed a sensitivity of $87 \%$ and specificity of $100 \%$ for distinguishing TDLs from gliomas or PCNSLs.' Another study showed that conventional MR imaging had a sensitivity of $81 \%$ and specificity of $57 \%$ for the differentiation of TDLs from high-grade gliomas. ${ }^{6}$

The diagnostic performance of an advanced MR imaging technique for the diagnosis of TDL was noted in 4 studies. ${ }^{9,12,14,21}$ In a comparison of TDLs with PCNSLs, 1 study demonstrated that the high $\mathrm{ADC}_{\text {minimum }}$ value with a threshold of $556 \times 10^{-6} \mathrm{~mm}^{2} / \mathrm{s}$ was the best indicator for differentiating TDLs from atypical PCNSLs (a sensitivity of $81 \%$ and specificity of $89 \%) .{ }^{12}$ In comparisons of TDLs with high-grade gliomas, 1 study using combined DTI and DSC perfusion showed a sen- 

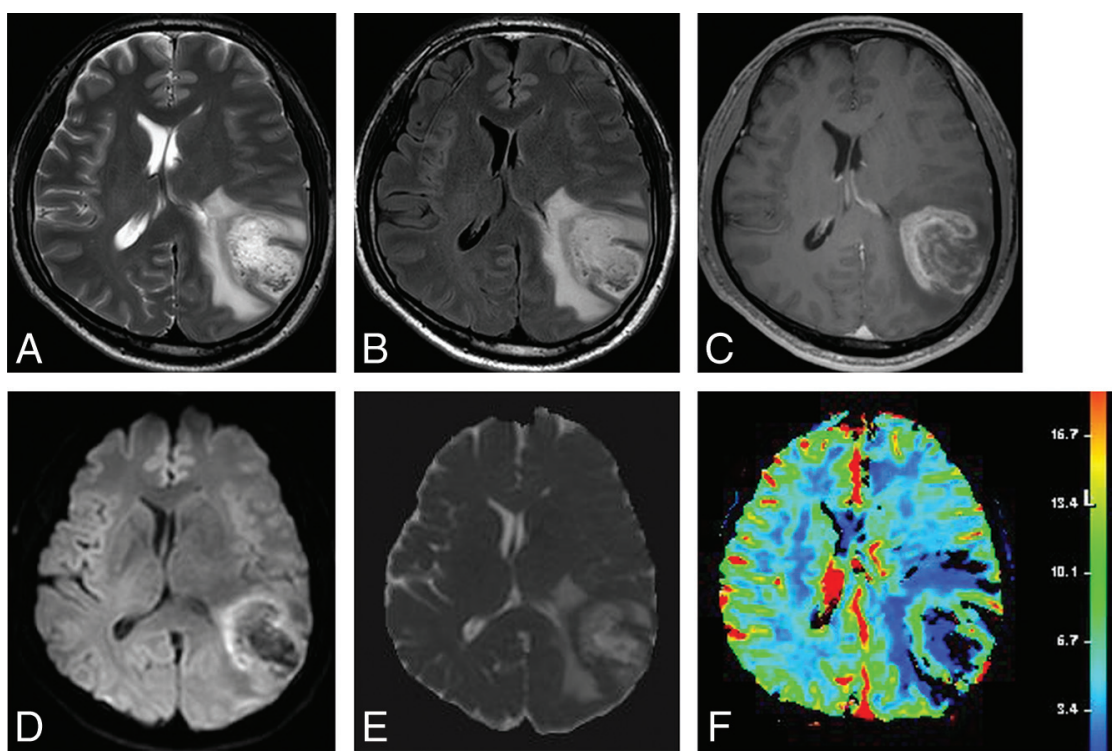

FIG 3. Images obtained in a 44-year-old man with biopsy-proved TDL. T2-weighted (A) and FLAIR $(B)$ images show a well-defined high-signal-intensity lesion in the left cerebral hemisphere with mild perilesional edema. $C$, Contrast-enhanced T1-weighted image shows open ring enhancement. DWI $(D)$ and the corresponding ADC map $(E)$ reveal high ADC within the lesion and peripheral restricted diffusion. $F$, DSC demonstrates low cerebral blood volume.

sitivity of $71 \%$ and specificity of $93 \%,{ }^{6}$ while another study using DTI showed a sensitivity of $92 \%$ and specificity of $88 \% .^{21}$ Two studies revealed that the combination of conventional MR imaging and advanced MR imaging improved the diagnostic performance. ${ }^{6,14}$

\section{Diagnostic Performance of MR Imaging for the Diagnosis of TDLs: Meta-Analysis}

Five original articles evaluated the overall diagnostic performance of MR imaging for differentiating a TDL from primary brain tumor. ${ }^{6,9,12,14,21}$ The individual sensitivities and specificities were $67 \%-91 \%$ and $79 \%-100 \%$. The pooled sensitivity was $89 \%$ (95\% CI, 82\%-93\%), and the pooled specificity was $94 \%$ (95\% CI, 89\%-97\%; Fig 4). The area under the hierarchic summary receiver operating characteristic curve was 0.93 (95\% CI, 0.90-0.95; On-line Fig 2). There was no heterogeneity in the sensitivity $\left(\mathrm{I}^{2}=0.0 \%\right)$ or specificity $\left(\mathrm{I}^{2}=45.72 \%\right)$. The Deeks funnel plot demonstrated that the likelihood of publication bias was low $(P=.48$, On-line Fig 3).

\section{DISCUSSION}

The current study reports the conventional MR imaging findings for TDLs, covering 19 studies with 476 patients. The pooled incidence of open ring or incomplete rim enhancement was 35\% (95\% CI, 24\%-47\%). In addition, the pooled incidences of a T2 hypointense rim, absent or mild mass effect, and absent or mild perilesional edema were $48 \%$ (95\% CI, 25\%-68\%), 67\% (95\% CI, $42 \%-87 \%$ ), and $57 \%(95 \% \mathrm{CI}, 27 \%-82 \%)$, respectively. The overall diagnostic performance of MR imaging for differentiating TDL from primary brain tumor demonstrated a pooled sensitivity of $89 \%(95 \%$ CI, 82\%-93\%) and a pooled specificity of $94 \%(95 \%$ CI, 89\%-97\%). Open ring or incomplete rim enhancement showed a high specificity (98\%-100\%). Therefore, conventional MR imaging findings can be of help in the accurate diagnosis of
TDL. In addition, our work could prove useful to the literature and may prompt the conduct of prospective case collections or consortial work.

In routine clinical practice, the differentiation of TDL from primary brain tumor is difficult. Open ring or incomplete rim enhancement as a conventional MR imaging finding was described as highly specific (94\%) for atypical brain demyelination in 32 illustrated cases identified on a MEDLINE search. ${ }^{35}$ The present study demonstrated a pooled incidence of open ring or incomplete rim enhancement of 35\% (95\% CI, 24\%-47\%); however, a high specificity $(98 \%-100 \%)$ was noted. In addition, the pooled incidence of open ring or incomplete rim enhancement was significantly higher than the incidence of closed ring or complete rim enhancement (18\% [95\% CI, 11\%-29\%]). The enhancing area of the ring is regarded as representing the leading edge of demyelination and therefore favors the white matter side of the lesion. ${ }^{36}$ Open ring or incomplete rim enhancement may be useful for differentiating a TDL from primary brain tumor.

The differentiation of a TDL from a PCNSL can sometimes be challenging, clinically, radiologically, and even pathologically. A few studies have tried to differentiate TDLs from PCNSLs using advanced MR imaging techniques. Two studies demonstrated a significantly higher $\mathrm{ADC}_{\text {minimum }}$ in TDL than in PCNSL, ${ }^{12,14}$ with 1 study demonstrating that $\mathrm{ADC}_{\text {minimum }}$ with a threshold of $556 \times 10^{-6} \mathrm{~mm}^{2} / \mathrm{s}$ was the best indicator for differentiating TDL from atypical PCNSL (a sensitivity of $81 \%$ and specificity of $89 \%) .{ }^{12}$ Histologically, TDLs may demonstrate peripheral active breakdown of myelin and a dense inflammatory infiltrate consisting of activated macrophages. ${ }^{37}$ Another study showed a lower choline/NAA ratio in TDLs than in PCNSLs, with a threshold for differentiation of 1.73 (a sensitivity of $89 \%$ and specificity of $76 \%) .{ }^{38}$ In addition, noncontrast CT hypoattenuation of MR imaging-enhanced regions was observed in $93 \%$ of TDL cases, but only $4 \%$ of primary brain tumors. ${ }^{9}$ One study revealed that the combination of conventional MR imaging and advanced MR imaging improved the diagnostic performance for differentiating TDL from PCNSLs or high-grade gliomas. ${ }^{14}$ ADC values, MR spectroscopy, and noncontrast CT may help in diagnosing TDLs; however, further study is required to determine the added value of advanced MR imaging techniques in the differentiation of TDLs from PCNSLs.

A previous article reported that most patients with TDLs showed an excellent response to corticosteroid treatment, with a substantial decrease in lesion size or disappearance on follow-up imaging. ${ }^{25}$ One of the studies included in the present meta-analysis reported that all patients with TDLs received corticosteroid treatment after surgical biopsy and showed no evidence of recurrence or radiologic aggravation during the 4.2 years of the follow- 


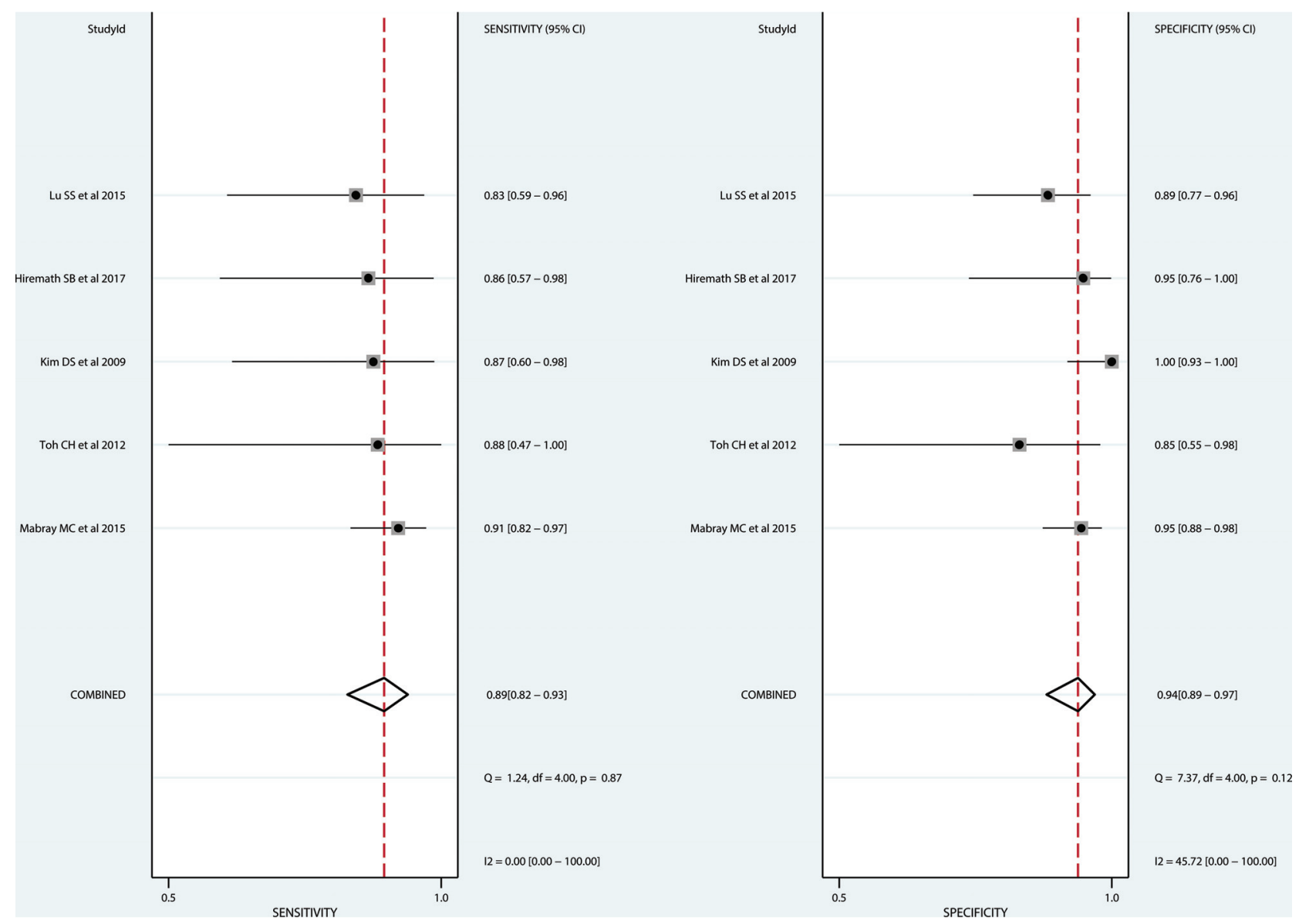

FIG 4. Coupled forest plots of the sensitivity and specificity of MR Imaging for the diagnosis of a TDL. Numbers are estimates with $95 \%$ confidence intervals in parentheses.

up. ${ }^{9}$ Other studies revealed that $35 \%-62 \%$ of patients with TDLs had a monophasic course and were diagnosed with a clinically isolated syndrome. ${ }^{4,8}$ A correlation between MR imaging findings and treatment response in patients with TDL has not yet been established, and further study on this may be needed.

This study has several limitations. First, 17 of the 19 studies were retrospective observational studies with a small sample size, and there were only 5 studies differentiating a TDL from primary brain tumor. However, the included studies represented the full extent of the currently available evidence. Our study may prompt prospective studies or consortial work. Second, heterogeneity was noted for conventional MR imaging findings. We therefore performed meta-regression to explore the effects of heterogeneity but found no covariates. Conventional MR imaging findings are inherently subjective, and the small samples due to the rarity of TDLs may affect the heterogeneity. Third, it is unclear how many of the included patients were positive for aquaporin 4 antibodies or myelin oliogodendrocyte glycoprotein antibodies and whether this influences MR imaging findings in TDLs. To overcome these limitations, we performed our systematic review and meta-analysis using recent robust methodology, including hierarchic logistic regression modeling, ${ }^{29-31}$ and reported our findings according to the following guidelines: Preferred Reporting Items for Systematic Reviews and Meta-Analyses, ${ }^{28}$ the Handbook for Diagnostic Test Accuracy Reviews published by the Cochrane Collaboration, ${ }^{39}$ and the Agency for Health Care Research and Quality. ${ }^{40}$

\section{CONCLUSIONS}

Conventional MR imaging findings may help in the accurate diagnosis of TDLs. However, further study is required to determine the added value of advanced MR imaging techniques in the differentiation of a TDL from primary brain tumor.

\section{REFERENCES}

1. Hardy TA, Chataway J. Tumefactive demyelination: an approach to diagnosis and management. J Neurol Neurosurg Psychiatry 2013;84: 1047-53 CrossRef Medline

2. Ayrignac X, Menjot de Champfleur N, Menjot de Champfleur S, et al. Brain magnetic resonance imaging helps to differentiate atypical multiple sclerosis with cavitary lesions and vanishing white matter disease. Eur J Neurol 2016;23:995-1000 CrossRef Medline

3. Wallner-Blazek M, Rovira A, Fillipp M, et al. Atypical idiopathic inflammatory demyelinating lesions: prognostic implications and relation to multiple sclerosis. J Neurol 2013;260:2016-22 CrossRef Medline

4. Altintas A, Petek B, Isik N, et al. Clinical and radiological characteristics of tumefactive demyelinating lesions: follow-up study. Mult Scler 2012;18:1448-53 CrossRef Medline

5. Cha S, Pierce S, Knopp EA, et al. Dynamic contrast-enhanced T2*weighted MR imaging of tumefactive demyelinating lesions. AJNR Am J Neuroradiol 2001;22:1109-16 Medline

6. Hiremath SB, Muraleedharan A, Kumar S, et al. Combining diffusion tensor metrics and DSC perfusion imaging: can it improve the diagnostic accuracy in differentiating tumefactive demyelination from high-grade glioma? AJNR Am J Neuroradiol 2017;38:685-90 CrossRef Medline 
7. Jain RS, Khan I, Kandelwal K, et al. Tumefactive demyelinating lesions (TDLs): a case series of clinicoradiological features. Clin Neurol Neurosurg 2017;162:91-94 CrossRef Medline

8. Kilic AK, Kurne AT, Oguz KK, et al. Mass lesions in the brain: tumor or multiple sclerosis? Clinical and imaging characteristics and course from a single reference center. Turk Neurosurg 2013;23: 728-35 CrossRef Medline Medline

9. Kim DS, Na DG, Kim KH, et al. Distinguishing tumefactive demyelinating lesions from glioma or central nervous system lymphoma: added value of unenhanced $C T$ compared with conventional contrastenhanced MR imaging. Radiology 2009;251:467-75 CrossRef Medline

10. Kiriyama T, Kataoka H, Taoka T, et al. Characteristic neuroimaging in patients with tumefactive demyelinating lesions exceeding 30 mm. J Neuroimaging 2011;21:e69-77 CrossRef Medline

11. Kuan YC, Wang KC, Yuan WH, et al. Tumefactive multiple sclerosis in Taiwan. PLoS One 2013;8:e69919 CrossRef Medline

12. Lu SS, Kim SJ, Kim N, et al. Histogram analysis of apparent diffusion coefficient maps for differentiating primary CNS lymphomas from tumefactive demyelinating lesions. AJR Am J Roentgenol 2015; 204:827-34 CrossRef Medline

13. Lucchinetti CF, Gavrilova RH, Metz I, et al. Clinical and radiographic spectrum of pathologically confirmed tumefactive multiple sclerosis. Brain 2008;131:1759-75 CrossRef Medline

14. Mabray MC, Cohen BA, Villanueva-Meyer JE, et al. Performance of apparent diffusion coefficient values and conventional MRI features in differentiating tumefactive demyelinating lesions from primary brain neoplasms. AJR Am J Roentgenol 2015;205:1075-85 CrossRef Medline

15. Malhotra HS, Jain KK, Agarwal A, et al. Characterization of tumefactive demyelinating lesions using MR imaging and in-vivo proton MR spectroscopy. Mult Scler 2009;15:193-203 CrossRef Medline

16. Miron S, Tal S, Achiron A. Diffusion tensor imaging analysis of tumefactive giant brain lesions in multiple sclerosis. J Neuroimaging 2013;23:453-59 CrossRef Medline

17. Qi W, Jia GE, Wang X, et al. Cerebral tumefactive demyelinating lesions. Oncol Lett 2015;10:1763-68 CrossRef Medline

18. Saini J, Chatterjee S, Thomas B, et al. Conventional and advanced magnetic resonance imaging in tumefactive demyelination. Acta Radiol 2011;52:1159-68 CrossRef Medline

19. Sánchez P, Meca-Lallana V, Barbosa A, et al. Tumefactive demyelinating lesions of 15 patients: clinico-radiological features, management and review of the literature. J Neurol Sci 2017;381:32-38 CrossRef Medline

20. Siri A, Carra-Dalliere C, Ayrignac X, et al. Isolated tumefactive demyelinating lesions: diagnosis and long-term evolution of 16 patients in a multicentric study. J Neurol 2015;262:1637-45 CrossRef Medline

21. Toh CH, Wei KC, Ng SH, et al. Differentiation of tumefactive demyelinating lesions from high-grade gliomas with the use of diffusion tensor imaging. AJNR Am J Neuroradiol 2012;33:846-51 CrossRef Medline

22. Yiu EM, Laughlin S, Verhey LH, et al; Canadian Pediatric Demyelinating Disease Network. Clinical and magnetic resonance imaging (MRI) distinctions between tumefactive demyelination and brain tumors in children. J Child Neurol 2014;29:654-65 CrossRef Medline

23. Given CA 2nd, Stevens BS, Lee C. The MRI appearance of tumefactive demyelinating lesions. AJR Am J Roentgenol 2004;182:195-99 CrossRef Medline
24. Seewann A, Enzinger C, Filippi M, et al. MRI characteristics of atypical idiopathic inflammatory demyelinating lesions of the brain: a review of reported findings. J Neurol 2008;255:1-10 CrossRef Medline

25. Kepes JJ. Large focal tumor-like demyelinating lesions of the brain: intermediate entity between multiple sclerosis and acute disseminated encephalomyelitis? A study of 31 patients. Ann Neurol 1993; 33:18-27 CrossRef Medline

26. Dagher AP, Smirniotopoulos J. Tumefactive demyelinating lesions. Neuroradiology 1996;38:560-65 CrossRef Medline

27. Whiting PF, Rutjes AW, Westwood ME, et al; QUADAS-2 Group. QUADAS-2: a revised tool for the quality assessment of diagnostic accuracy studies. Ann Intern Med 2011;155:529-36 CrossRef Medline

28. Liberati A, Altman DG, Tetzlaff J, et al. The PRISMA statement for reporting systematic reviews and meta-analyses of studies that evaluate health care interventions: explanation and elaboration. Ann Intern Med 2009;151:W65-94 Medline

29. Suh $\mathrm{CH}$, Park SH. Successful publication of systematic review and meta-analysis of studies evaluating diagnostic test accuracy. Korean J Radiol 2016;17:5-6 CrossRef Medline

30. Kim KW, Lee J, Choi SH, et al. Systematic review and meta-analysis of studies evaluating diagnostic test accuracy: a practical review for clinical researchers, Part I: general guidance and tips. Korean J Radiol 2015;16:1175-87 CrossRef Medline

31. Lee J, Kim KW, Choi SH, et al. Systematic review and meta-analysis of studies evaluating diagnostic test accuracy: a practical review for clinical researchers, Part II: statistical methods of meta-analysis. Korean J Radiol 2015;16:1188-96 CrossRef Medline

32. Higgins JP, Thompson SG, Deeks JJ, et al. Measuring inconsistency in meta-analyses. BMJ 2003;327:557-60 CrossRef Medline

33. Egger M, Davey Smith G, Schneider M, et al. Bias in meta-analysis detected by a simple, graphical test. BMJ 1997;315:629-34 CrossRef Medline

34. Deeks JJ, Macaskill P, Irwig L. The performance of tests of publication bias and other sample size effects in systematic reviews of diagnostic test accuracy was assessed. J Clin Epidemiol 2005;58:882-93 CrossRef Medline

35. Masdeu JC, Quinto C, Olivera C, et al. Open-ring imaging sign: highly specific for atypical brain demyelination. Neurology 2000;54: 1427-33 CrossRef Medline

36. He J, Grossman RI, Ge Y, et al. Enhancing patterns in multiple sclerosis: evolution and persistence. AJNR Am J Neuroradiol 2001; 22:664-69 Medline

37. Lassmann $\mathrm{H}$. The pathologic substrate of magnetic resonance alterations in multiple sclerosis. Neuroimaging Clin N Am 2008;18:56376, ix CrossRef Medline

38. Lu SS, Kim SJ, Kim HS, et al. Utility of proton MR spectroscopy for differentiating typical and atypical primary central nervous system lymphomas from tumefactive demyelinating lesions. AJNR Am J Neuroradiol 2014;35:270-77 CrossRef Medline

39. Deeks JJ, Bossuyt PM, Gatsonis C, eds. 2013 Cochrane Handbook for DTA Reviews, Version 1.0.0. The Cochrane Collaboration. http:// srdta.cochrane.org/handbook-dta-reviews. Accessed October 9, 2017

40. Trikalinos TA, Balion CM, Coleman CI, et al. Chapter 8: meta-analysis of test performance when there is a "gold standard." J Gen Intern Med 2012;27(Suppl 1):S56-66 CrossRef Medline 